\title{
Thermal Effects on Modal Properties of Dual-Core Yb-Doped Fibers
}

\author{
Poli, Federica; Laegsgaard, Jesper; Cucinotta, Annamaria; Selleri, Stefano
}

Published in:

Journal of Lightwave Technology

Link to article, DOI:

10.1109/JLT.2018.2886047

Publication date:

2018

Document Version

Peer reviewed version

Link back to DTU Orbit

Citation (APA):

Poli, F., Laegsgaard, J., Cucinotta, A., \& Selleri, S. (2018). Thermal Effects on Modal Properties of Dual-Core Yb-Doped Fibers. Journal of Lightwave Technology, 37(4), 1075 - 1083.

https://doi.org/10.1109/JLT.2018.2886047

\section{General rights}

Copyright and moral rights for the publications made accessible in the public portal are retained by the authors and/or other copyright owners and it is a condition of accessing publications that users recognise and abide by the legal requirements associated with these rights.

- Users may download and print one copy of any publication from the public portal for the purpose of private study or research.

- You may not further distribute the material or use it for any profit-making activity or commercial gain

- You may freely distribute the URL identifying the publication in the public portal

If you believe that this document breaches copyright please contact us providing details, and we will remove access to the work immediately and investigate your claim. 


\title{
Thermal Effects on Modal Properties of Dual-Core Yb-Doped Fibers
}

\author{
Federica Poli, Member, IEEE, Jesper Lægsgaard, Annamaria Cucinotta, Member, IEEE, \\ and Stefano Selleri, Senior Member, IEEE
}

\begin{abstract}
The impact of thermo-optic refractive-index changes arising from the core heat load in a dual-core fiber amplifier is studied through finite-element based numerical simulations. Trends in coupling lengths, effective area, core overlaps of fundamental and higher-order supermodes, effects of asymmetric heat loads, and thermo-optic mode coupling parameters are quantified. It is concluded that the coupling between cores is only moderately altered by the overall heat load, but can be strongly sensitive to asymmetric loads. The influence of the core coupling strength is demonstrated to be very important on the supermode effective area, besides the shrinking due to thermal effects. While the thermo-optic index perturbations can lead to guidance of higher-order supermodes, it seems realistic to maintain single-mode operation through gain suppression of the higher-order modes. On the other hand, thermo-optic couplings between fundamental supermodes are found to be quite strong.
\end{abstract}

\section{INTRODUCTION}

$\mathbf{L}$ arge-core Yb-doped fiber amplifiers are central components in fiber-based high-power laser systems, which are becoming increasingly important in a range of industrial processing applications [1], [2]. Especially for short-pulsed (nanosecond or shorter) lasers core area scaling is crucial to suppress detrimental effects of Raman scattering and selfphase modulation. The use of very large cores makes it difficult to maintain single-mode operation, especially because excess heat from the amplification process generates additional refractive-index gradients around the core through the thermooptic effect [3]-[5]. The presence of higher-order modes in turn leads to limitations from dynamic thermo-optic instabilities, which currently constitute the main limitation for power scaling of short-pulsed fiber lasers [6]-[10].

A possible route to further power scaling is to incorporate a number of amplifying cores in each fiber [11]-[13]. The individual cores may then be kept sufficiently small to maintain single-mode guidance, whereas the total core area could in principle be very large. The fiber as a whole will of course still be multimode, but the coupling between different cores can be reduced by increasing the core separation.

So far, most theoretical efforts on thermo-optic effects in fiber amplifiers have been directed towards understanding thermally induced mode profile changes [3]-[5], [14] and

F. Poli, A. Cucinotta and S. Selleri are with the Department of Engineering and Architecture, University of Parma, I-43124 Parma, Italy, e-mail: federica.poli@unipr.it.

J. Lægsgaard is with DTU Fotonik, Department of Photonics Engineering, Technical University of Denmark, DK-2800 Kongens Lyngby, Denmark.

Copyright (c) 2015 IEEE. Personal use of this material is permitted. However, permission to use this material for any other purposes must be obtained from the IEEE by sending a request to pubs-permissions@ieee.org. mode couplings [6]-[10], [15], [16] in single-core amplifiers. Thermo-optic mode couplings in a dual-core fiber were recently investigated numerically [17], but considering only the fundamental modes guided in the absence of a thermal load, and without taking thermal mode deformations into account. Clearly, there is an immediate need to assess the validity of such an approach by studying the influence of static heat loads on the eigenmodes of dual- and multi-core amplifiers.

While the modal properties of a passive array of cores are well understood, limited research into thermo-optic perturbations of such modes has been performed until now [18]. Several pertinent questions need to be answered for optimizing fiber design. How will the thermo-optic effects influence coupling lengths? What will be the impact of power asymmetries between cores? Will the coupling strength between the cores influence the effective area shrinking due to thermal effects? Will the thermal load lead to guidance of higher-order modes, and will such modes be problematic with respect to amplification or thermo-optic instabilities?

The present paper aims to provide some answers to these questions through numerical simulation studies of the simplest possible multicore fiber, namely the dual-core fiber. We will consider a structure with intrinsically single-mode cores, and study the evolution of the fundamental supermodes, as well as the higher-order modes, as the heat load on the cores is increased. It will be shown that thermo-optic effects increase coupling lengths for large core separations, but decrease them for close-lying cores. It is further demonstrated that both the core center-to-center distance as well as the heat load strongly impact the supermode effective areas. Moreover, it is shown that higher-order modes may become guided through the
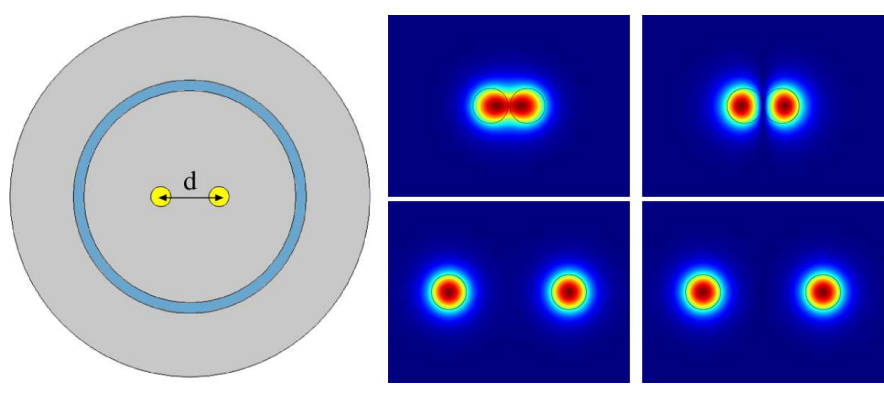

Fig. 1. (Left) Cross-section of the dual-core fiber analyzed for different centerto-center distance $d$ between two doped cores (yellow regions). The light-blue ring is the air layer surrounding the inner cladding. (Right) Electric field modulus distribution of fundamental even and odd SM for (top) $d=20 \mu \mathrm{m}$ and (bottom) $d=65 \mu \mathrm{m}$. 


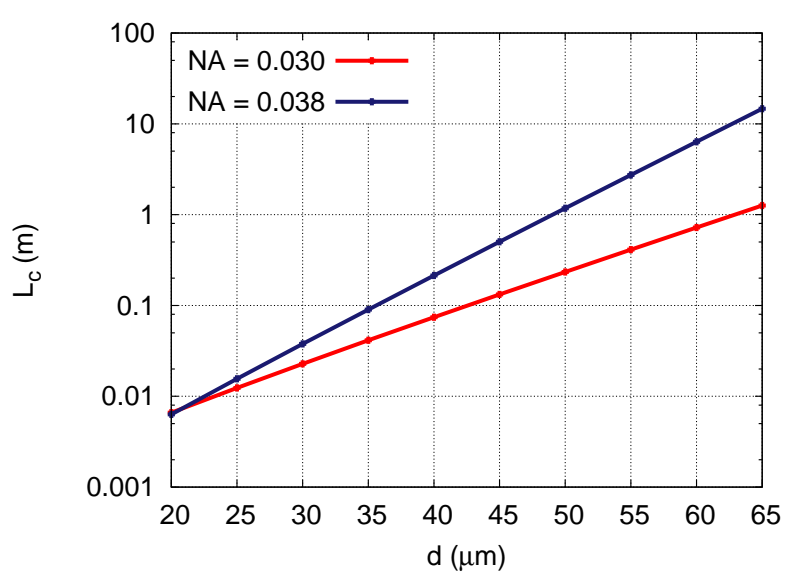

Fig. 2. Coupling length $L_{c}$ between fundamental even and odd SMs as a function of distance $d$ for dual-core fiber with different $N A$.

thermo-optic effect, however for increasing core separations, there is a substantial gain suppression of these modes relative to the fundamental modes, even at very large heat loads. Finally, their thermo-optic couplings to the fundamental modes are found to be about three times smaller than those calculated in comparable single-core fibers.

\section{MOdELING OF DUAL-CORE FIBERS}

The cross-section of the dual-core fiber considered for the numerical analysis is shown in Fig. 1(left). Each of the Ybdoped step-index cores, represented by the yellow regions, have a diameter of $19 \mu \mathrm{m}$ [12]. Two numerical aperture $N A$ values of 0.03 and 0.038 have been considered, corresponding to $V$-parameter values of 1.73 and 2.20 , respectively, at the signal wavelength of $1032 \mathrm{~nm}$. Thus only the fundamentalmode (FM) even and odd Supermodes (SMs) are guided at this wavelength, which we choose to consider since it is the one experimentally used with the multicore fiber in [12]. The distance $d$ between the core centers has been varied from 20 $\mu \mathrm{m}$ to $65 \mu \mathrm{m}$, in order to investigate different optical coupling regimes between the cores. A silica region, with diameter of $200 \mu \mathrm{m}$ and refractive index calculated according to the Sellmeier equation [19], surrounded by a $10 \mu \mathrm{m}$ wide air layer, shown as the light-blue region in Fig. 1(left), act as the pump cladding in the dual-core fiber, whose outer diameter is 340 $\mu \mathrm{m}$.

SMs at $1032 \mathrm{~nm}$ in dual-core fibers with different $d$ values have been calculated with a full-vector modal solver based on the Finite Element Method (FEM) [20]. The electric field modulus distribution of fundamental even and odd SMs, respectively, is reported in Fig. 1(right), from top to bottom, for the fiber with $d=20 \mu \mathrm{m}$ and with $d=65 \mu \mathrm{m}$. The effect of the heat load generated by the quantum defect on the guiding properties of even and odd SMs has been analyzed using a FEM-based solver with an embedded thermal model [14]. This method has been already successfully applied to study the heating influence on guided modes and single-mode regime of different kinds of large mode area photonic crystal fibers with a single $\mathrm{Yb}$-doped core [14]. The model has been recently generalized to consider the generation of heat load in
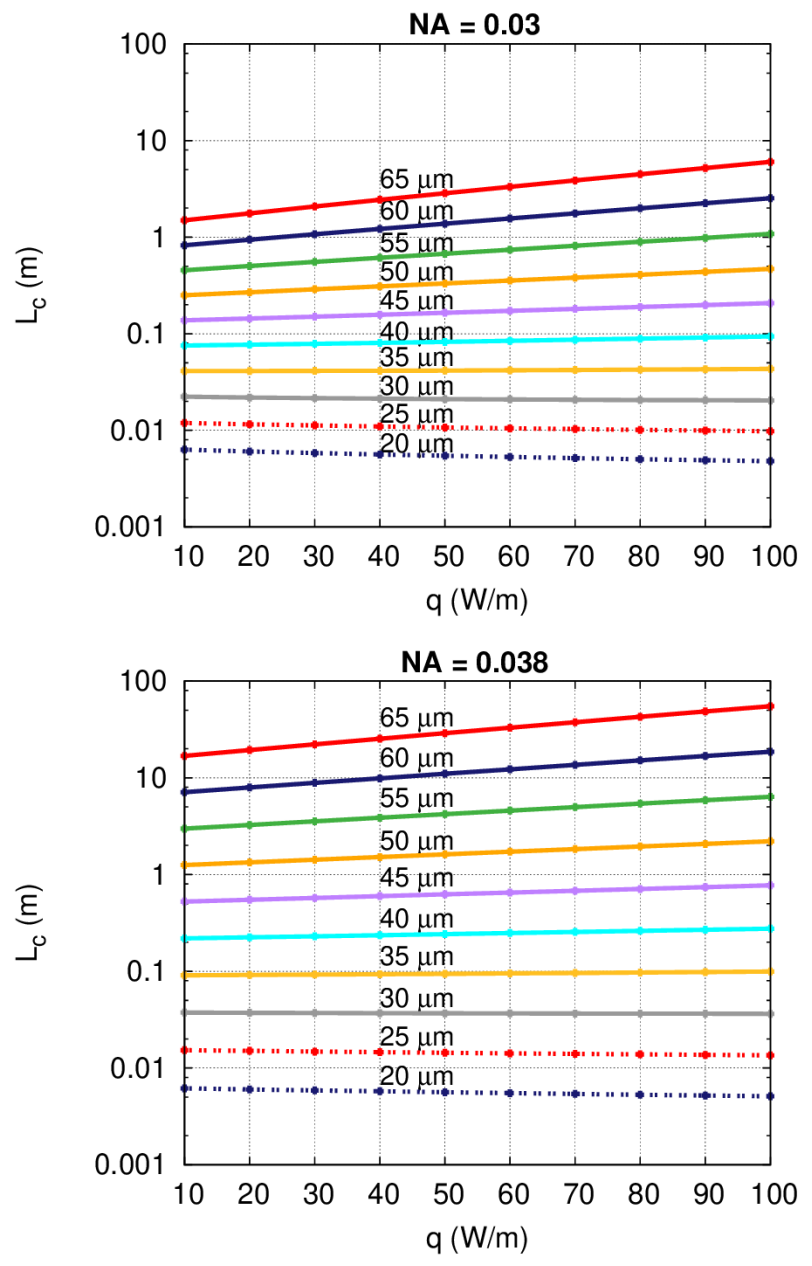

Fig. 3. Coupling length $L_{c}$ between fundamental even and odd SMs as a function of heat load $q$ for different distance $d$ when (top) $N A=0.03$ and (bottom) $N A=0.038$.

more than one $\mathrm{Yb}$-doped region of a multi-core fiber crosssection [18]. In the present analysis we apply it to the dualcore fiber case. In particular, we consider a uniform heat load $q$ across each core, and quantify it in terms of its cross-sectional integral, i.e. in units of $\mathrm{W} / \mathrm{m}$. Note also that the $q$ value given in the following is for a single core, so that the total heat load on the fiber is two times larger. The steady-state heat equation has been solved to calculate the temperature gradient $\Delta T$ on the dual-core fiber cross-section, taking into account the silica thermal conductivity $\kappa=1.38 \mathrm{~W} /(\mathrm{m} \cdot \mathrm{K})$ and the heat dissipation at the outer fiber edge with a convective heat transfer coefficient $h=80 \mathrm{~W} /\left(\mathrm{m}^{2} \cdot \mathrm{K}\right)$. Due to the thermooptic effect, a refractive index change $\Delta n=\eta \Delta T$, with $\eta$ $=1.16 \cdot 10^{-5} \mathrm{~K}^{-1}$ the thermo-optic coefficient of silica, is induced in the dual-core fiber cross-section [21]. In singlecore fibers the thermally-induced refractive index profile has a parabolic shape in the core, with a maximum in the center, and a logarithmic decay in the cladding [14]. The behaviour of $\Delta n$ on multi-core fiber cross-section is more complex and depends strictly on the number of cores and on their geometric configuration [18]. In the present analysis the effect of the thermally-induced $\Delta n$ on the coupling between fundamental 

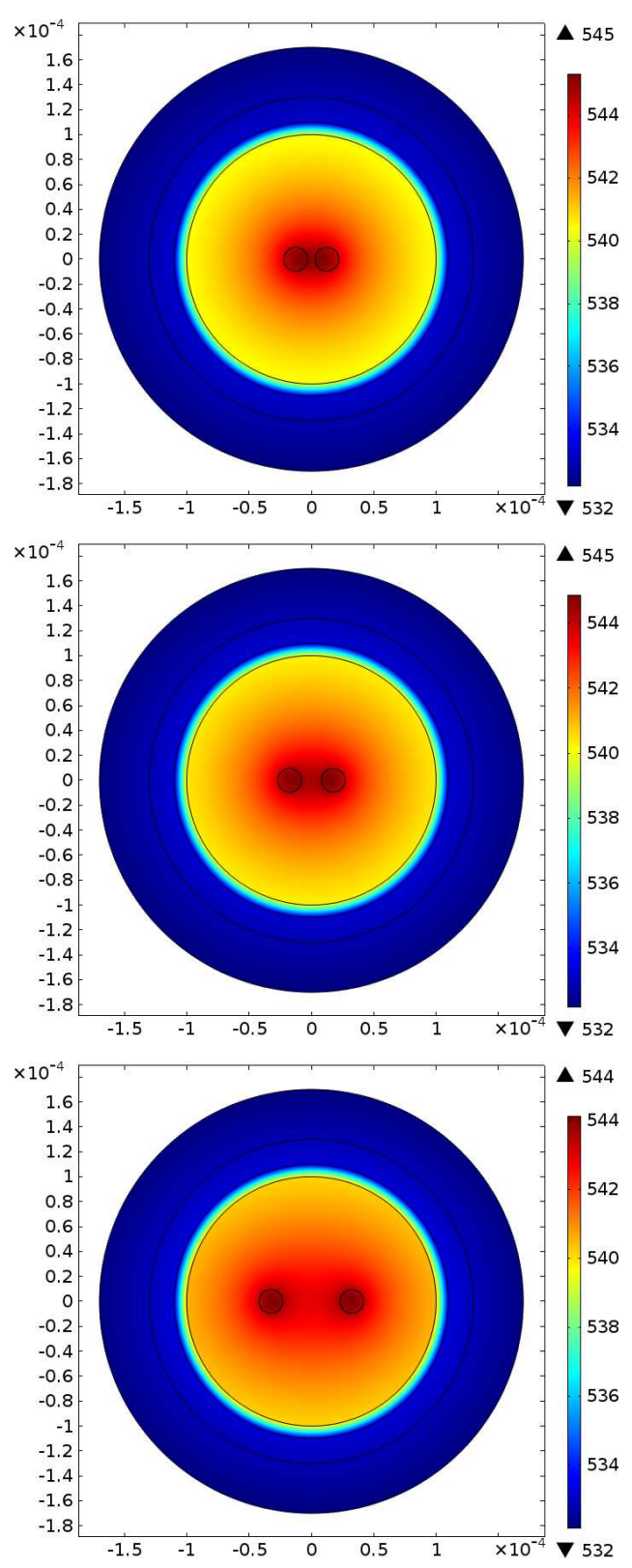

Fig. 4. Temperature distribution on the dual-core fiber cross-section when $q$ $=10 \mathrm{~W} / \mathrm{m}$ is applied in both cores, for (top) $d=25 \mu \mathrm{m}$, (center) $d=35 \mu \mathrm{m}$ and (bottom) $d=65 \mu \mathrm{m}$.

SMs, as well as on the dual-core fiber single-mode regime has been investigated. In particular, the coupling length $L_{c}=\frac{\pi}{\Delta \beta}$ between fundamental even and odd SMs and their effective area, and the overlap integral of both fundamental and higherorder SMs on one of the doped core [22] have been calculated for different heat load values in the range between $10 \mathrm{~W} / \mathrm{m}$ and $100 \mathrm{~W} / \mathrm{m}$.

\section{RESULTS AND DISCUSSION}

\section{A. Fundamental supermode coupling}

As shown in Fig. 2, the coupling length between fundamental even and odd SMs strongly depends on the distance $d$ for dual-core fibers with both the $N A$ values considered

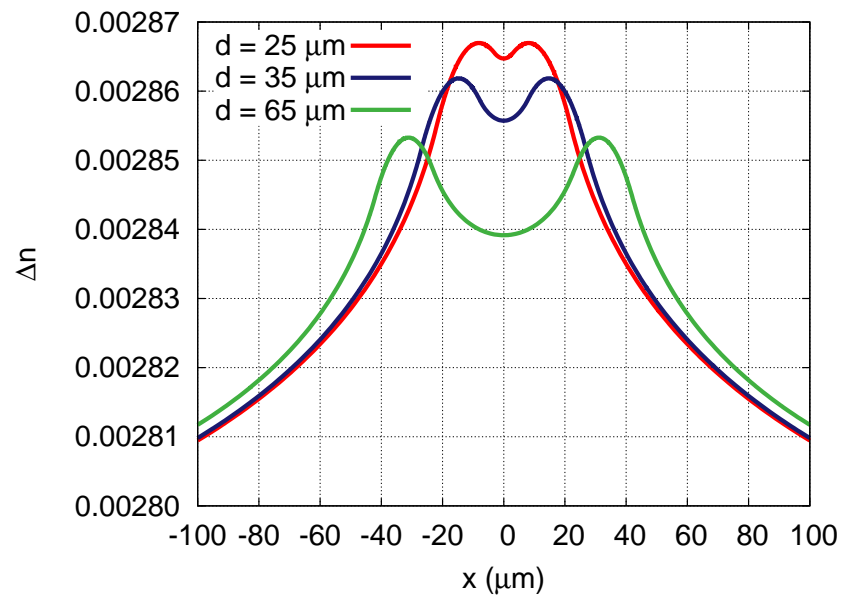

Fig. 5. Thermally-induced refractive index change $\Delta n$ in the pump cladding along the horizontal line crossing the core centers of dual-core fibers with different $d$ values when $q=10 \mathrm{~W} / \mathrm{m}$ is applied in both cores.

in the analysis. For the lowest $N A, L_{c}$ is as short as 0.66 $\mathrm{cm}$ when the cores are at the closest distance $d=20 \mu \mathrm{m}$, while it increases to $1.26 \mathrm{~m}$ as the core separation reaches the maximum value of $d=65 \mu \mathrm{m}$. Notice that the effect of the $N A$ increase to 0.038 becomes significant only at large core distance. In fact, for $d=65 \mu \mathrm{m}$ the coupling length difference reaches a maximum of $13 \mathrm{~m}$, corresponding to a tenfold increase of $L_{c}$ when going from $N A=0.03$ to $N A$ $=0.038$. On the contrary, the $L_{c}$ difference is negligible at the shortest core separations. This demonstrates that, in the latter condition, the coupling between the two fundamental supermodes is so strong that it is not significantly affected by the rise of the core refractive index, corresponding to the NA increase.

When the heat load $q$ is applied to both the cores, the coupling length $L_{c}$ for dual-core fibers with $N A=0.03$ and $N A=0.038$ significantly changes, as shown in Fig. 3(top) and (bottom), respectively. It is important to underline that the thermal load effect is different according to the core separation. In particular, when the distance $d$ is short, that is $d \leq 25 \mu \mathrm{m}, L_{c}$ gets slightly shorter as $q$ increases from $10 \mathrm{~W} / \mathrm{m}$ to $100 \mathrm{~W} / \mathrm{m}$. For example, when $d=20 \mu \mathrm{m}, L_{c}$ is around $0.63 \mathrm{~cm}$ for $q=10 \mathrm{~W} / \mathrm{m}$ and it becomes $0.48 \mathrm{~cm}$ for the highest heat load value. In this condition, the two cores are so close that the temperature increase due to the heat load is significant also in the thin silica region between them, as demonstrated by Fig. 4(top), where the temperature distribution on the transverse section of the dual-core fiber with $d=25 \mu \mathrm{m}$ for $q=10 \mathrm{~W} / \mathrm{m}$ is reported. As a consequence, the refractive-index barrier between the cores is reduced and the coupling between the two single-core waveguides becomes even stronger. This is clearly shown by the thermally-induced $\Delta n$ in the pump cladding along the horizontal line crossing the two core centers of the fiber with $d=25 \mu \mathrm{m}$, reported in Fig. 5 for an applied heat load of $10 \mathrm{~W} / \mathrm{m}$. In fact the $\Delta n$ maximum along this direction, that is 0.0028670 , is reached near to the inner boundary of each core, around $8 \mu \mathrm{m}$ far from the fiber cross-section center, and not in the central position. Moreover, 


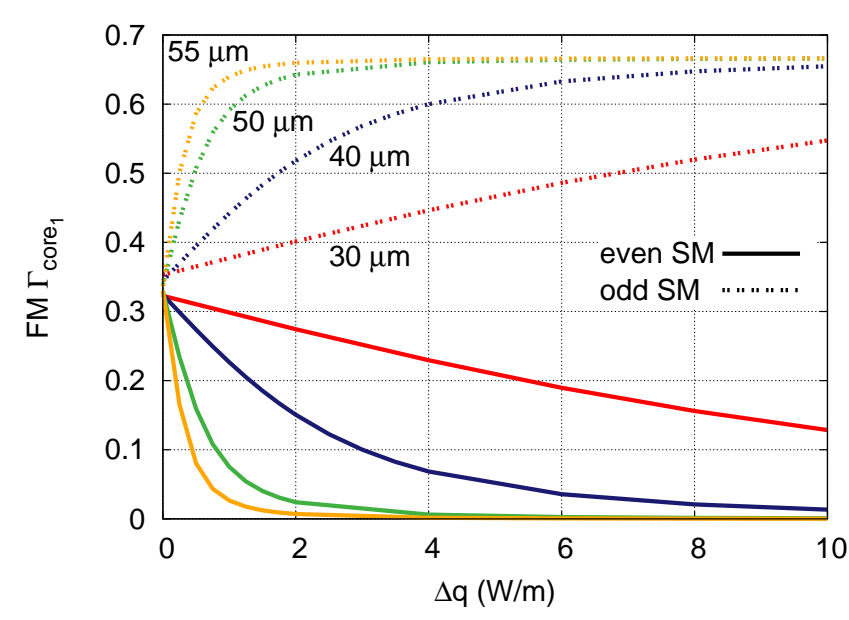

Fig. 6. Overlap integral of fundamental even and odd SMs in core 1 as a function of heat load difference $\Delta q$, applied only to second core, for different distance $d$ values when $q=10 \mathrm{~W} / \mathrm{m}$ and $N A=0.03$.

in between the two cores $\Delta n$ reaches a minimum value, that is 0.0028647 , which is only $2.3 \cdot 10^{-6}$ lower than the maximum one. Notice that the opposite behaviour is obtained for large core-to-core distance, that is for $d \geq 40 \mu \mathrm{m}$. In this situation the $L_{c}$ increase with the applied $q$ is higher as $d$ becomes wider. In fact the temperature increase due to the applied heat load is significant mainly inside the boundary of each core, as demonstrated by Fig. 4(bottom) for the fiber with $d=65 \mu \mathrm{m}$ when $q=10 \mathrm{~W} / \mathrm{m}$. Consequently, the effect of the thermallyinduced $\Delta n$ contributes to reduce the coupling between the fundamental even and odd SMs. This is because the thermally induced refractive-index increase in each core enhances mode confinement, thereby reducing the mode overlap between cores. As shown in Fig. 5 for $q=10 \mathrm{~W} / \mathrm{m}$, when the core separation increases to $d=65 \mu \mathrm{m}$ in the dual-core fiber, the difference between $\Delta n$ maximum and minimum value in the region between the cores increases to $1.4 \cdot 10^{-5}$, being the maximum position almost coincident with the core center. This is still a relatively small perturbation compared to the core-cladding refractive-index difference, which is $\sim 3 \cdot 10^{-4}$ for $N A=0.03$, and $\sim 5 \cdot 10^{-4}$ for $N A=0.038$. However, the thermal gradients scale linearly with the heat load, and for $q=$ $100 \mathrm{~W} / \mathrm{m}$ the thermal index perturbations are thus comparable in magnitude to the index variations of the cold fiber. As a result, there are significant modifications of the mode profiles at heat loads of this magnitude.

It is interesting to notice that an intermediate regime exists for a distance $d$ between $30 \mu \mathrm{m}$ and $35 \mu \mathrm{m}$, where $L_{c}$ is almost unaffected by the heat load applied to the two doped cores. In fact, as $q$ increases from $10 \mathrm{~W} / \mathrm{m}$ to $100 \mathrm{~W} / \mathrm{m}$, the coupling length is reduced of $8.5 \%$ when $d=30 \mu \mathrm{m}$, starting from a value of about $2.23 \mathrm{~cm}$. In the same way, when $d=$ $35 \mu \mathrm{m}$, only a $5 \%$ widening of the initial value of $4.12 \mathrm{~cm}$ is obtained. The temperature distribution and the corresponding thermally-induced $\Delta n$ obtained for the dual-core fiber with $d=$ $35 \mu \mathrm{m}$ when the applied heat-load is $q=10 \mathrm{~W} / \mathrm{m}$ are reported, respectively, in the central panel of Fig. 4 and in Fig. 5. As shown in Fig. 3(bottom), an analogous behavior is obtained
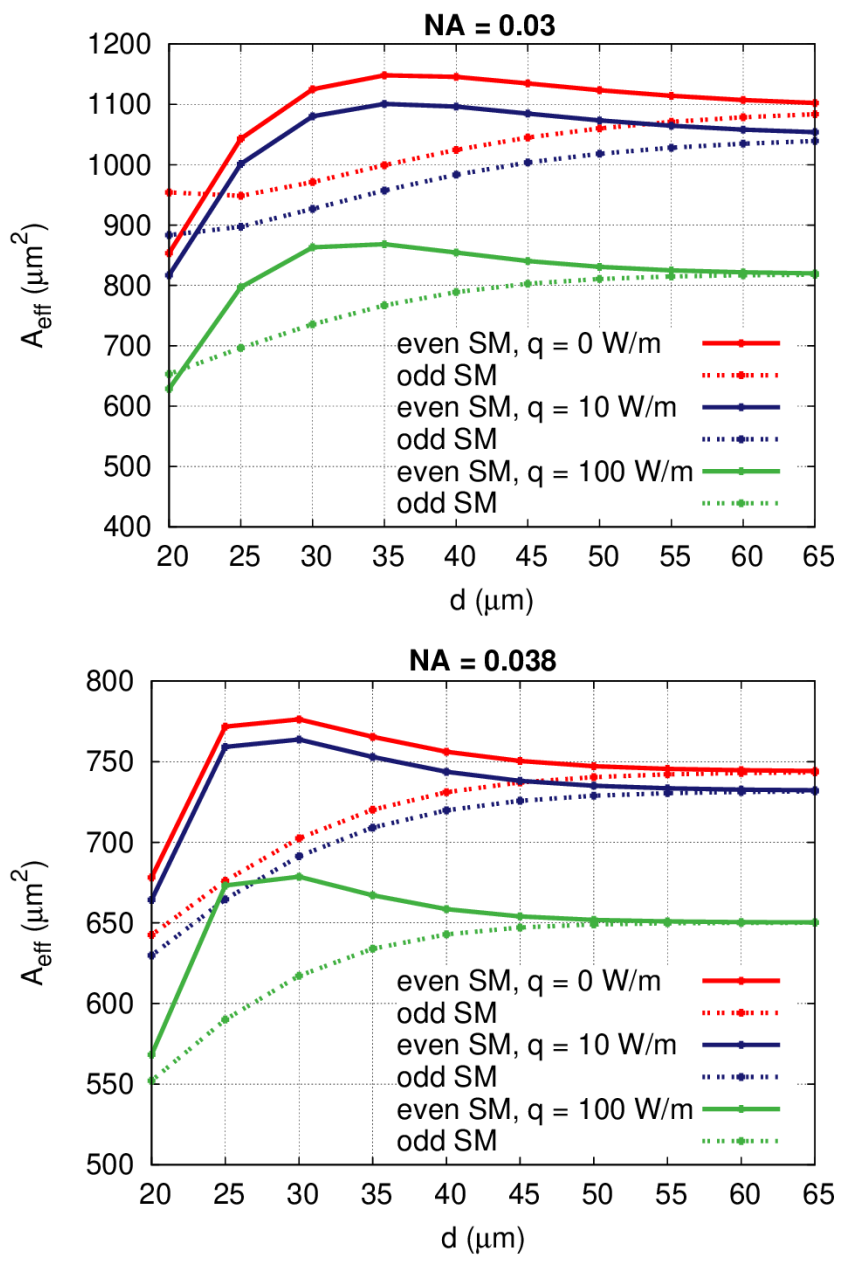

Fig. 7. Effective area of fundamental even and odd SM as a function of distance $d$ for $q=0,10$ and $100 \mathrm{~W} / \mathrm{m}$ applied to each core when (top) $N A$ $=0.03$ and (bottom) $N A=0.038$.

for the dual-core fibers with the higher $N A$ value. Notice that, when $d$ is between $30 \mu \mathrm{m}$ and $35 \mu \mathrm{m}$, the absolute value of the relative $L_{c}$ change caused by the heat load increase up to $100 \mathrm{~W} / \mathrm{m}$ is still lower than $10 \%$, being $2.9 \%$ and $9.4 \%$, respectively, for the core separation corresponding to the lower and the upper edge of the range. The main difference with respect to the dual-core fiber with $N A=0.03$ is a general increase of the coupling length between fundamental even and odd SMs for a fixed value of the core distance. For example, $L_{c}$ higher than $1 \mathrm{~m}$, which is a typical length for high-power fiber amplifiers, is obtained when the core separation is $50 \mu \mathrm{m}$ for all the considered $q$ values. On the contrary, it is necessary to apply a heat load of at least $30 \mathrm{~W} / \mathrm{m}$ in a fiber with $d$ $=60 \mu \mathrm{m}$ to reach the same coupling condition if the lower numerical aperture is considered. Finally, results reported in Fig. 3 demonstrate that, for a certain heat load $q$, the influence of the core center-to-center distance on the fundamental SM coupling is more significant in the dual-core fiber with $N A=$ 0.038, as shown in Fig. 2 in the absence of thermal effects. 

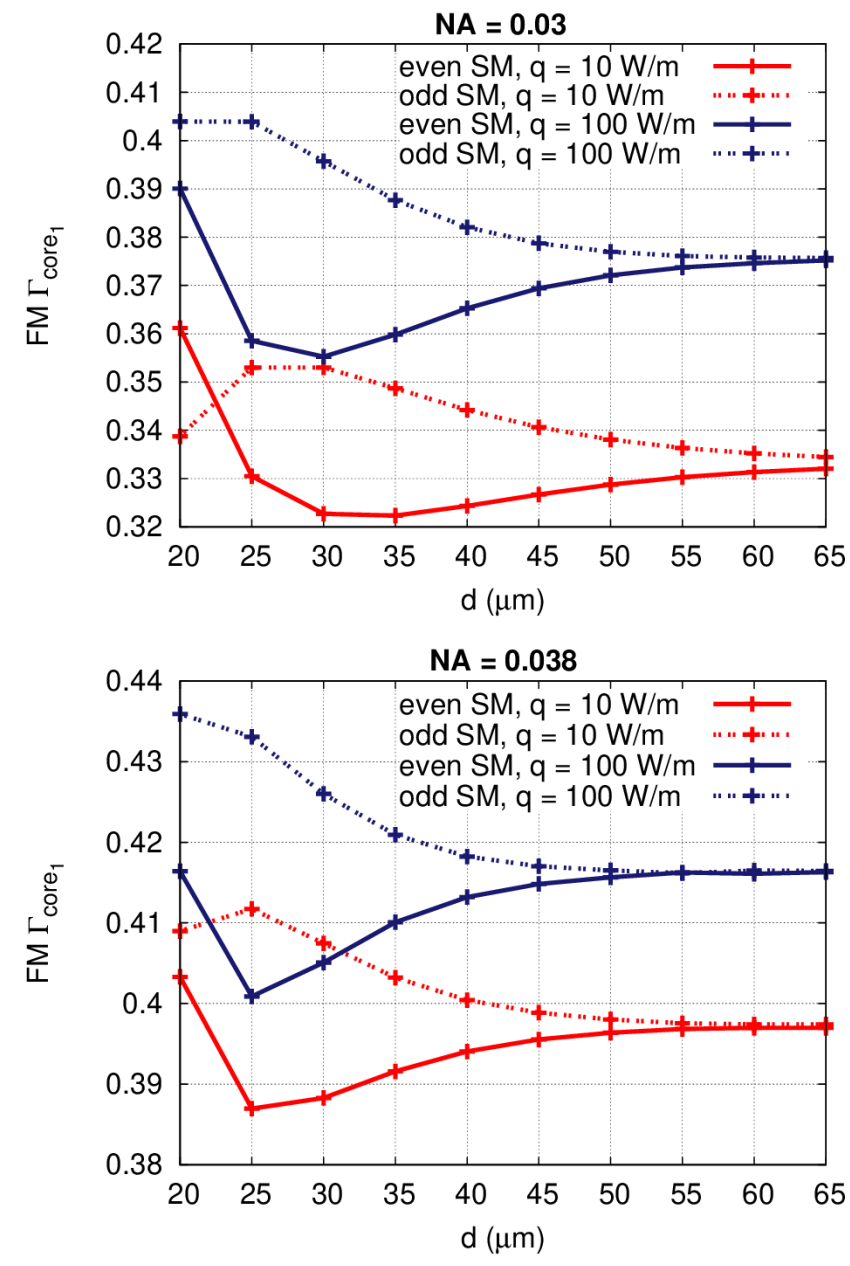

Fig. 8. Core overlap integral of fundamental even and odd SM as a function of distance $d$ for $q=10,100 \mathrm{~W} / \mathrm{m}$ applied to each core when (top) $N A=$ 0.03 and (bottom) $N A=0.038$.

\section{B. Supermode coupling with asymmetric heat load}

The strength of the coupling between the two Yb-doped cores in the fiber amplifier can be evaluated also by analyzing the effect on fundamental SMs of an asymmetry in the applied heat load, represented by $\Delta q$. This is relevant because it may not always be possible in practice to ensure a perfectly equal signal power into both cores. Also the onset of dynamic thermo-optic instabilities can lead to a power imbalance between the cores. The resulting asymmetric refractive-index perturbation can lead to a decoupling of the cores, with the supermode profiles reverting to the guided-mode profiles of the individual cores. We consider $q$ and $q+\Delta q$ in the left core (core 1) and in the right one (core 2) respectively, with $q=10 \mathrm{~W} / \mathrm{m}$. The overlap integral $\Gamma_{\text {core } 1}$ of the fundamental even and odd SM field on core 1 has been calculated as a function of $\Delta q$ in the range $0 \div 10 \mathrm{~W} / \mathrm{m}$ for fibers with $N A=0.03$ and different core separation, that is with $d=30$, 40, 50 and $55 \mu \mathrm{m}$. As reported in Fig. 6, for large distance between the two cores the asymmetry in the refractive index profile, due to the heat load difference, significantly affects the supermodes even at low $\Delta q$. In fact, $\Gamma_{\text {core } 1}$, which has almost the same value around 0.33 for both the SMs in the
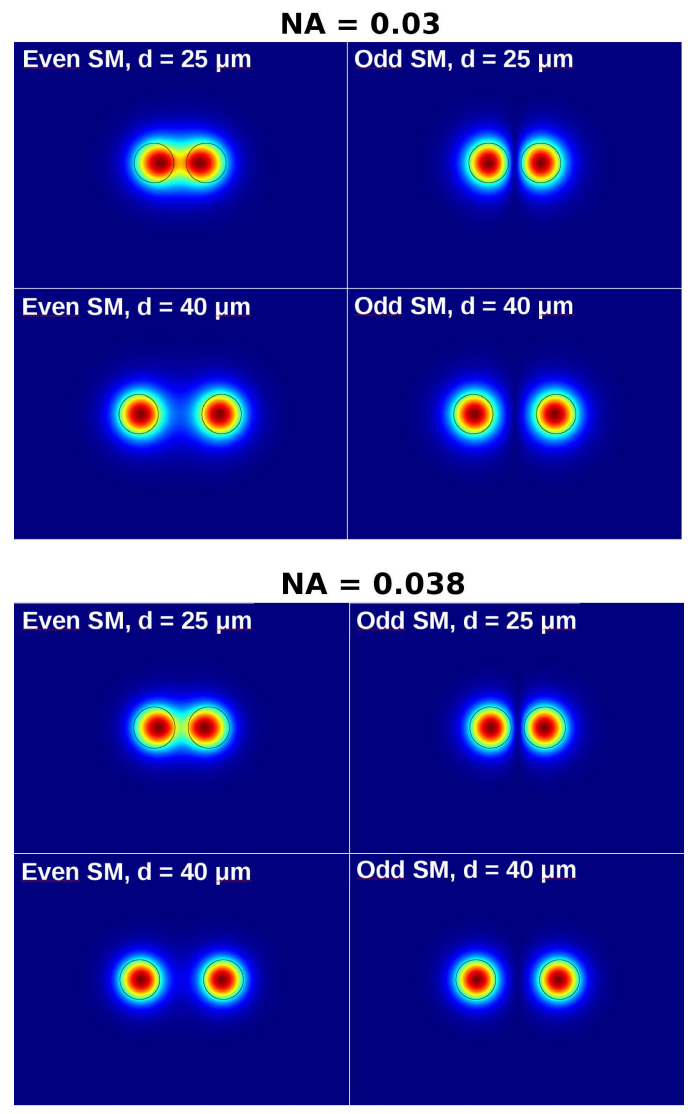

Fig. 9. Fundamental SM electric field modulus distribution for $d=25 \mu \mathrm{m}$ and $d=40 \mu \mathrm{m}$ obtained when $q=100 \mathrm{~W} / \mathrm{m}$ is applied to each core, for (top) $N A=0.03$ and (bottom) $N A=0.038$.

symmetric heat load situation, has a significant increase for the odd supermode and, at the same time, becomes negligible for the even one as $\Delta q$ increases. In particular, the condition $\Gamma_{\text {core } 1} \leq 0.05$ for the even SM and $\Gamma_{\text {core } 1} \geq 0.6$ for the odd one is obtained when $\Delta q \geq 1.25 \mathrm{~W} / \mathrm{m}$ and $\Delta q \geq 0.75 \mathrm{~W} / \mathrm{m}$ for $d=50 \mu \mathrm{m}$ and $55 \mu \mathrm{m}$, respectively. This means that, as $\Delta q$ increases, the even and odd SMs are replaced by the singlecore eigenmodes, localized in the separate cores. Notice that the same effect is reached in the fiber with $40 \mu \mathrm{m}$ core-tocore distance for a higher $\Delta q$ value, that is around $4 \mathrm{~W} / \mathrm{m}$. Finally, results demonstrate that for the dual-core fiber with $d=30 \mu \mathrm{m}$ the coupling between the cores is so strong that, even by doubling the applied heat load to core 2, it is still possible to identify SMs rather than single-core eigenmodes. In this situation, that is for $\Delta q=10 \mathrm{~W} / \mathrm{m}, \Gamma_{\text {core } 1}$ is 0.13 and 0.53 for the fundamental even and odd SM, respectively.

\section{Effective area}

Thermal effects have a remarkable impact also on the guided mode effective area $A_{e f f}$, which shrinks as the heat load increases [14]. This can negatively affect the threshold of nonlinear effects, thus reducing the advantages provided by multicore fiber designs. As a consequence, it is important to evaluate the $A_{\text {eff }}$ behaviour for the fundamental even and odd SM of the dual-core fibers as a function of the heat load $q$ by taking into account different $d$ values, which correspond to 

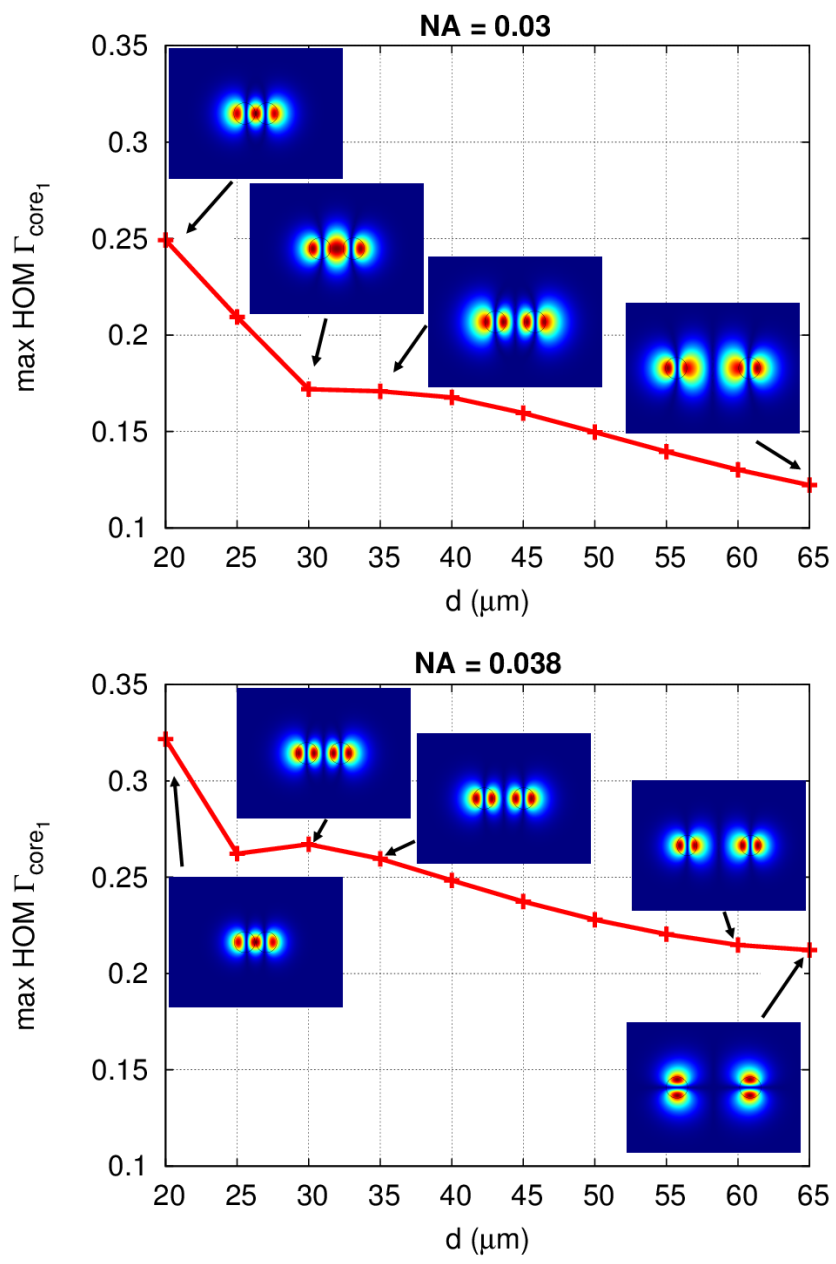

Fig. 10. Maximum core overlap integral of first higher-order SM as a function of distance $d$ for $q=100 \mathrm{~W} / \mathrm{m}$ applied to each core when (top) $N A=0.03$ and (bottom) $N A=0.038$. Insets: electric field modulus distribution of the considered HOM.

different coupling strengths between the two cores. Fig. 7(top) shows the effective area obtained for the fundamental SMs of the two-core fibers with $N A=0.03$ when no heat load is applied and when $q=10 \mathrm{~W} / \mathrm{m}$ and $100 \mathrm{~W} / \mathrm{m}$. Notice that, as expected looking at the field distributions, the odd SM $A_{\text {eff }}$ is lower than the even supermode one for all the considered $d$ values, except for $d=20 \mu \mathrm{m}$, in the absence of thermal effects. Moreover, the difference between the $A_{\text {eff }}$ values of the two SMs decreases as the core center-to-center distance enlarges, showing a convergence towards twice the effective area value of the FM of the single-core fiber with the same $N A$, that is $547 \mu \mathrm{m}^{2}$. It is interesting to underline that the even SM $A_{e f f}$ is lower than the single-core fiber limit when the distance between the cores is reduced, that is for $d=20$ $\mu \mathrm{m}$ and $25 \mu \mathrm{m}$. In particular, the effective area of the even SM has a significant increase for $d$ in the range between 20 $\mu \mathrm{m}$ and $35 \mu \mathrm{m}$, reaching a maximum value of $1148 \mu \mathrm{m}^{2}$, then it slowly decreases, when the distance between the cores becomes wider. A similar $A_{e f f}$ behaviour is obtained when the heat load is applied to both the cores. In particular, the curves of the even and odd SM effective area converge to twice the value obtained for the FM of the single-core fiber when the same $q$ is considered. Notice also that the maximum value of the even SM effective area becomes lower as $q$ increases, being $1100 \mu \mathrm{m}^{2}$ and $868 \mu \mathrm{m}^{2}$ for $d=35 \mu \mathrm{m}$ when $q=10$ $\mathrm{W} / \mathrm{m}$ and $100 \mathrm{~W} / \mathrm{m}$, respectively. Moreover, as the heat load becomes higher, the difference between the $A_{\text {eff }}$ values of the two fundamental supermodes vanishes for a smaller core separation. For example, when $q=100 \mathrm{~W} / \mathrm{m}$, the difference becomes negligible for $d=55 \mu \mathrm{m}$. Results of the effective area calculations for the dual-core fibers with $N A=0.038$ are shown in Fig. 7(bottom). It is important to underline that, as expected, lower $A_{\text {eff }}$ values are obtained for all $d$ values, with and without thermal effects, with respect to the analogous fibers with $N A=0.03$, due to the stronger field confinement in the core. If the heat load is applied to the doped fiber cores, an effective area decrease is obtained for both the fundamental SMs, which is lower that the one caused by $q$ in the fibers with $N A=0.03$. In particular, the maximum $A_{\text {eff }}$ of the even SM, which is $776 \mu \mathrm{m}^{2}$ for $d=30 \mu \mathrm{m}$ without heat load, becomes $764 \mu \mathrm{m}^{2}$ and $679 \mu \mathrm{m}^{2}$ when $q=10 \mathrm{~W} / \mathrm{m}$ and $100 \mathrm{~W} / \mathrm{m}$, respectively. Finally, in the dual-core fibers with higher $N A$ the difference between the fundamental SM $A_{\text {eff }}$ values becomes negligible for a core center-to-center distance around $50 \mu \mathrm{m}$ and $45 \mu \mathrm{m}$ for an applied heat load of $10 \mathrm{~W} / \mathrm{m}$ and $100 \mathrm{~W} / \mathrm{m}$, respectively. In summary, results demonstrate that the $N A$ increase gives the same qualitative effects on the effective area, that is a decrease and an early convergence of the even and odd SM values, obtained when the heat load $q$ becomes higher, since the thermal load effectively increases the dual-core fiber $N A$.

\section{Single-mode regime}

The thermally-induced refractive index change, generated in the doped core as a side effect of the optical amplification process, has a potentially significant impact on the singlemode properties of the fiber, by favoring the confinement also of the first Higher-Order Mode (HOM) [14]. As it has been demonstrated for single-core fiber amplifiers, the interaction of the HOM with the fundamental mode can lead to the detrimental phenomenon of transverse mode instability [6], [7], [15], [16]. In the present analysis the effect of the heat load $q$ on the single-mode regime of dual-core fibers has been studied by calculating the overlap integral $\Gamma_{\text {core } 1}$ for fundamental and higher-order even and odd supermodes.

Fig. 8(top) shows $\Gamma_{\text {core } 1}$ values for the fundamental SMs as a function of the core separation in the dual-core fiber with $N A=0.03$ when $q=10 \mathrm{~W} / \mathrm{m}$ and $100 \mathrm{~W} / \mathrm{m}$. As expected, the overlap integral is higher when the heat load increases, due to the stronger field confinement in the doped core. Notice that, for the highest applied $q, \Gamma_{\text {core } 1}$ of the fundamental odd SM is greater than that of the even mode, regardless of the core separation. This is due to the different field distribution of the two modes, especially in the silica region between the cores. As demonstrated by Fig. 9(top), the field intensity in this area is significant for the even SM, especially at small core separation, while being almost zero for the odd mode. The difference between $\Gamma_{\text {core } 1}$ values of the two fundamental SMs is maximal for the fiber with $d=30 \mu \mathrm{m}$ and $d=25 \mu \mathrm{m}$ for $q$ 


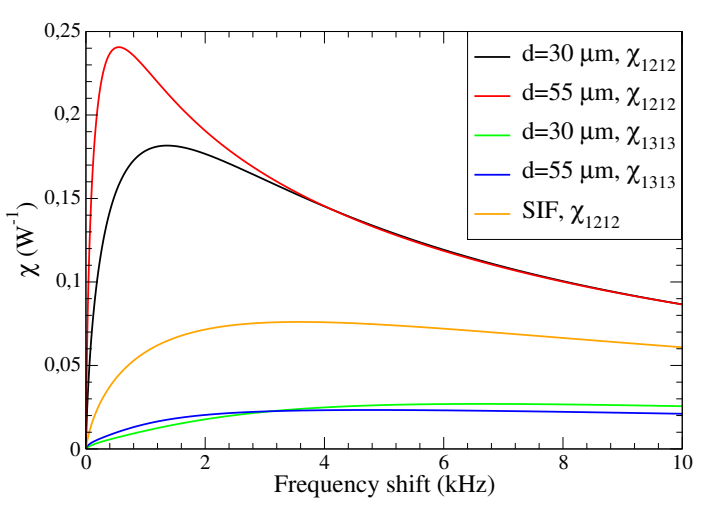

Fig. 11. Thermo-optic mode-coupling coefficients for NA=0.038 dual-core and single-core fibers at a total heat load of $200 \mathrm{~W} / \mathrm{m}$.

$=10 \mathrm{~W} / \mathrm{m}$ and $q=100 \mathrm{~W} / \mathrm{m}$, respectively, and it vanishes as $d$ becomes larger. Results reported in Fig. 8(bottom) demonstrate that the $\Gamma_{\text {core } 1}$ behaviour is similar, with slightly higher values for both the considered heat loads, for the dual-core fibers with $N A=0.038$. As expected, Fig. 9(bottom) shows that the electric field modulus of both the fundamental SMs is more tightly confined in the core due to the $N A$ increase from 0.03 to 0.038 .

The first group of higher-order supermodes in the dualcore fiber consists of four spatial mode distributions, or eight modes in total when different polarizations are counted as well [23]. In Fig. 10(top) $\Gamma_{\text {core } 1}$ values for fibers with $N A=$ 0.03 , obtained for $q=100 \mathrm{~W} / \mathrm{m}$, are reported as a function of the core separation for the mode with the highest core overlap integral. It is important to underline that the highest heat load provides the best situation for the higher-order supermode propagation and thus the worst one for maintaining the dual-core fiber single-mode behaviour. As shown by the field distribution insets, the higher-order supermode with the tighter confinement in the doped core is the even one with modal field aligned with the horizontal axis for $d$ between 20 $\mu \mathrm{m}$ and $30 \mu \mathrm{m}$, and the odd one with horizontally aligned field when the core separation becomes wider. Notice that the higher-order SM $\Gamma_{\text {core } 1}$ decreases from 0.25 to 0.12 as the core-to-core distance increases from $d=20 \mu \mathrm{m}$ and $d=65 \mu \mathrm{m}$. On the contrary, the overlap integral of the fundamental even SM obtained with the same applied heat load is less affected by the distance $d$ between the cores, being 0.39 and 0.375 for the fiber with the lowest and the highest core separation, respectively, as shown in Fig. 8(top). As a consequence, the difference between fundamental and higher-order SM $\Gamma_{\text {core } 1}$ is lower for fibers with low $d$ values and becomes higher as the distance between the two $\mathrm{Yb}$-doped cores increases, being 0.14 and 0.255 for $d=20 \mu \mathrm{m}$ to $d=65 \mu \mathrm{m}$, respectively. When the $N A$ of the dual-core fibers increases to 0.038 , the maximum overlap integral of the higher-order SMs becomes higher, as shown in Fig. 10(bottom), being 0.32 and 0.21 for $d=20 \mu \mathrm{m}$ to $d=65 \mu \mathrm{m}$, respectively. Notice that, according to the results reported in Fig. 8(bottom), the $\Gamma_{\text {core } 1}$ difference with respect to the fundamental even SM slightly decreases to 0.096 and 0.206 . Finally, differently from the case of lower $N A$ dual-core fibers, the higher-order even supermode with horizontally aligned field has the greatest $\Gamma_{\text {core } 1}$ value only for $d \leq 25 \mu \mathrm{m}$. Moreover, the higher-order odd SM with field aligned with the vertical axis is the one with the highest confinement in the doped core for the dual-core fiber with largest $d$.

Due to the symmetry of the dual-core structure, the total overlap with the Yb-doped regions is two times $\Gamma_{\text {core } 1}$. For $N A=0.03$ the differential overlap between fundamental and HOM supermodes is then always larger than 0.3 [24], [25] for $d \geq 30 \mu \mathrm{m}$, while for NA=0.038 the same can be said for $d \geq 35 \mu \mathrm{m}$. Such a differential overlap would usually be adequate for gain suppression of the HOMs except for very special circumstances. It should be noted that $q=100$ $\mathrm{W} / \mathrm{m}$, which implies a total heat load of $200 \mathrm{~W} / \mathrm{m}$, is a very high value. The quantum defect of $\mathrm{Yb}$ is typically less than $10 \%$ for $976 \mathrm{~nm}$ pumping, and is only 5.7\% for the signal wavelength of $1032 \mathrm{~nm}$ considered here. A heat load of 200 $\mathrm{W} / \mathrm{m}$ thus corresponds to the extraction of several $\mathrm{kW}$ per meter, which is a somewhat exceptional situation for presentday amplifier configurations. The existence of a substantial differential gain disfavoring the HOM supermodes even at such a high thermal load gives confidence that the HOM gain will also be suppressed at more realistic $q$ values.

\section{E. Thermo-optic interactions}

Apart from gain overlap integrals, the importance of higherorder modes can also be quantified through their thermooptic coupling to the fundamental supermodes. A convenient formal framework for this has been provided by Hansen $e t$ al. [15], who in a perturbative coupled-mode formalism related mode coupling between modes $m$ and $n$ to four-field overlap integrals of the form

$$
\begin{aligned}
\chi_{m n m n}(\Omega)= & \frac{\eta k_{0}}{\kappa n_{0}}\left(\frac{\lambda_{s}}{\lambda_{p}}-1\right) \operatorname{Im}\left[\int d \mathbf{r}_{\perp} \Psi_{m}\left(\mathbf{r}_{\perp}\right) \Psi_{n}\left(\mathbf{r}_{\perp}\right)\right. \\
& \left.\int_{d} d \mathbf{r}_{\perp}^{\prime} G\left(\mathbf{r}_{\perp}, \mathbf{r}_{\perp}^{\prime}, \Omega\right) \Psi_{m}\left(\mathbf{r}_{\perp}^{\prime}\right) \Psi_{n}\left(\mathbf{r}_{\perp}^{\prime}\right)\right] . \quad \text { (1) }
\end{aligned}
$$

Here $\lambda_{s}, \lambda_{p}$ are signal and pump wavelengths, $n_{0}$ is the silica refractive index and $k_{0}$ is the vacuum wave vector of the signal. The Greens function $G\left(\mathbf{r}_{\perp}, \mathbf{r}_{\perp}^{\prime}, \Omega\right)$ describes the temperature change at transverse coordinate $\mathbf{r}_{\perp}$ arising from a steady-periodic heat load at $\mathbf{r}_{\perp}^{\prime}$ with angular frequency $\Omega$. The $d$ subscript on the $\mathbf{r}_{\perp}^{\prime}$ integral indicates that this integral is only taken over the doped core regions where heat is generated. $\Psi_{m}$ and $\Psi_{n}$ are the (scalar) transverse profiles of the two coupled modes. When the $\chi(\Omega)$ function between two modes is known, the power fraction $\xi$ transferred to the higher-order mode through the thermo-optic nonlinearity can be estimated as in [15].

In Fig. $11 \chi$ functions between different modes in a dualcore amplifier are plotted. Modes 1 and 2 are the fundamental even and odd supermodes, whereas mode 3 is the higher-order mode with the largest core overlap at a total heat load of $200 \mathrm{~W} / \mathrm{m}$. For comparison, the $\chi$ function for $\mathrm{LP}_{01}$ and $\mathrm{LP}_{11}$ modes in a single-core Step-Index Fiber (SIF) is also shown. In this case mode 1 is $\mathrm{LP}_{01}$ and mode 2 is $\mathrm{LP}_{11}$. It can be noted that the thermo-optic mode coupling between the dual-core 
supermodes is considerably stronger than the mode coupling in a single-core amplifier. On the other hand, the coupling between the even fundamental supermode and the higher-order mode in the dual-core amplifier is about 3 times smaller than the single-core $\mathrm{LP}_{01}-\mathrm{LP}_{11}$ couplings, and 6-7 times smaller than the coupling between fundamental supermodes.

It may seem surprising that the peak values of $\chi_{1212}$ increases with increasing $d$, but one should remember that it represents coupling of delocalized supermodes. To a good approximation we can write

$$
\begin{aligned}
& \Psi_{1}\left(\mathbf{r}_{\perp}\right)=\frac{1}{\sqrt{2}}\left[\Psi_{01}\left(\mathbf{r}_{\perp}-\mathbf{r}_{l}\right)+\Psi_{01}\left(\mathbf{r}_{\perp}-\mathbf{r}_{r}\right)\right] \\
& \Psi_{2}\left(\mathbf{r}_{\perp}\right)=\frac{1}{\sqrt{2}}\left[\Psi_{01}\left(\mathbf{r}_{\perp}-\mathbf{r}_{l}\right)-\Psi_{01}\left(\mathbf{r}_{\perp}-\mathbf{r}_{r}\right)\right]
\end{aligned}
$$

where $\Psi_{01}$ is the scalar profile of a single-core fundamental mode, and $\mathbf{r}_{l, r}$ are the transverse positions of the left and right cores. Inserting Eqs. (2), (3) into Eq. (1), and using the symmetry of the structure, we derive

$$
\begin{aligned}
& \chi_{1212}(\Omega)=\frac{\eta k_{0}}{2 \kappa n_{0}}\left(\frac{\lambda_{s}}{\lambda_{p}}-1\right) \operatorname{Im}\left[\int d \mathbf{r}_{\perp}\left|\Psi_{01}\left(\mathbf{r}_{\perp}\right)\right|^{2}\right. \\
& \int_{r^{\prime}<r_{d}} d \mathbf{r}_{\perp}^{\prime} G\left(\mathbf{r}_{\perp}, \mathbf{r}_{\perp}^{\prime}, \Omega\right)\left|\Psi_{01}\left(\mathbf{r}_{\perp}^{\prime}\right)\right|^{2}- \\
& \int d \mathbf{r}_{\perp}\left|\Psi_{01}\left(\mathbf{r}_{\perp}-\mathbf{R}\right)\right|^{2} \\
& \left.\int_{r^{\prime}<r_{d}} d \mathbf{r}_{\perp}^{\prime} G\left(\mathbf{r}_{\perp}, \mathbf{r}_{\perp}^{\prime}, \Omega\right)\left|\Psi_{01}\left(\mathbf{r}_{\perp}^{\prime}\right)\right|^{2}\right]
\end{aligned}
$$

where $\mathbf{R}$ is the vector separating the two core centers, and $r_{d}$ is the core doping radius. The first term represent thermooptic couplings inside a single core, the second term represents couplings between the cores, i.e. the influence of a thermal load in one core on the temperature change in the other. As the core separation increases, the second term decays, but the first is constant, and the total coupling increases. A more intuitive explanation is the following: $\chi_{1212}$ describes how an asymmetric heat load (arising from the odd symmetry of the product $\Psi_{1} \Psi_{2}$ ) leads to an asymmetric index distribution coupling the even and odd supermodes. If the cores are widely separated, an increased heat load in one core is primarily felt in this core, whereas for closely-spaced cores it is also felt in the other core. Therefore the asymmetric (odd) part of the index perturbation is stronger for widely separated cores.

The nonlinear mode coupling between the fundamental supermodes $\Psi_{1}, \Psi_{2}$ was recently studied numerically [17]. It was argued that the $\chi_{1212}$ coefficient controls the magnitude of mode couplings and instabilities for closely spaced cores. However, for widely separated cores a more elaborate analysis is needed, and eventually the coupling vanishes even if $\chi_{1212}$ remains large. This was shown to be crucially dependent on the vanishing of $\Delta \beta$, the propagation-constant difference, between even and odd supermodes, as $d$ becomes large. Couplings between the fundamental supermodes depicted in Fig. 9 and higher-order supermodes like the ones depicted in Fig. 10 have so far not been studied by nonlinear simulations. However, for this case the propagation-constant difference will remain finite even as the core separation tends to infinity. From the analysis of [17] it is therefore reasonable to suppose that this coupling can be adequately quantified by $\chi_{1313}$ and should therefore be strongly reduced compared to mode couplings in a multimode step-index fiber.

\section{CONCLUSION}

In conclusion, the impact of thermally induced refractiveindex changes on the eigenmodes of a dual-core fiber amplifier has been studied through numerical simulations. For large core separations, a thermal load in the cores leads to an increase in the coupling lengths, or in other words, a weakening of power transfer between the cores. On the other hand, for closely spaced cores, the heat load leads to a slight decrease of coupling lengths, and for core separations of $30-35 \mu \mathrm{m}$, the coupling length has very little variation with heat load. An asymmetric heat load generally leads to decoupling of the cores, in the sense that the symmetric and antisymmetric supermodes found for a symmetric heat load gradually turn into modes localized around one of the cores. The magnitude of the asymmetry needed for decoupling varies from $\sim 1 \mathrm{~W} / \mathrm{m}$ for a core separation of $55 \mu \mathrm{m}$ to $>10 \mathrm{~W} / \mathrm{m}$ for a separation of $30 \mu \mathrm{m}$. The core center-to-center distance, which determines the coupling strength between the cores, has an important impact also on the fundamental supermode effective area. In particular, when the distance is around 20-25 $\mu \mathrm{m}$, the coupling is very strong and the effective area of both the supermodes of the dual-core fiber is significantly lower than twice the fundamental mode area of the corresponding single-core fiber. As the core separation enlarges, there is a significant increase of the even supermode effective area towards a maximum value, reached when the core separation is around 30-35 $\mu \mathrm{m}$, followed by a slow decrease to the single-core fiber limit. When the heat load is applied to the doped cores, the supermode effective area values decrease, as expected, but the behaviour versus the core separation is almost unaffected by thermal effects. Simulation results further demonstrate that for strong heat loads, supermodes composed of higher-order modes become guided, even though the individual cores of the fiber are single-mode. However, the differential core overlap between fundamental and higher-order supermodes is almost always above 0.3 , which will in most cases lead to efficient suppression of the higher-order modes due to differential gain. Furthermore, thermo-optic mode coupling coefficients between fundamental and higher-order supermodes are found to be about four times smaller than those calculated between even and odd fundamental supermodes, and two times smaller than those calculated between $\mathrm{LP}_{01}$ and $\mathrm{LP}_{11}$ modes in a stepindex fiber with the same NA and mode area as the dualcore fiber. This implies, on the other hand, that thermo-optic couplings between even and odd fundamental supermodes are stronger than the couplings in the corresponding step-index fiber, which can lead to strong power fluctuations between the cores at relatively low average powers.

\section{FUNDING}

J. Lægsgaard acknowledges financial support from the University of Parma visiting professors program 2018. 


\section{REFERENCES}

[1] C. Jauregui, J. Limpert, and A. Tünnermann, "High-power fibre lasers," Nature Photonics, vol. 7, no. 11, pp. 861-867, Nov 2013.

[2] M. N. Zervas and C. A. Codemard, "High power fiber lasers: A review," IEEE Journal of Selected Topics in Quantum Electronics, vol. 20, no. 5, pp. 219-241, Sep 2014.

[3] S. Hädrich, T. Schreiber, T. Pertsch, J. Limpert, T. Peschel, R. Eberhardt, and A. Tünnermann, "Thermo-optical behavior of rare-earth-doped low-NA fibers in high power operation," Opt. Express, vol. 14, no. 13, pp. 6091-6097, Jun 2006. [Online]. Available: http://www.opticsexpress.org/abstract.cfm?URI=oe-14-13-6091

[4] E. Coscelli, F. Poli, T. T. Alkeskjold, M. M. Jorgensen, L. Leick, J. Broeng, A. Cucinotta, and S. Selleri, "Thermal effects on the single-mode regime of distributed modal filtering rod fiber," Journal of Lightwave Technology, vol. 30, no. 22, pp. 3494-3499, Nov 2012.

[5] M. M. Johansen, K. R. Hansen, M. Laurila, T. T. Alkeskjold, and J. Lægsgaard, "Estimating modal instability threshold for photonic crystal rod fiber amplifiers," Opt. Express, vol. 21, no. 13, pp. 15 409-15417, Jul 2013. [Online]. Available: http: //www.opticsexpress.org/abstract.cfm?URI=oe-21-13-15409

[6] T. Eidam, C. Wirth, C. Jauregui, F. Stutzki, F. Jansen, H.-J. Otto, O. Schmidt, T. Schreiber, J. Limpert, and A. Tünnermann, "Experimental observations of the threshold-like onset of mode instabilities in high power fiber amplifiers," Opt. Express, vol. 19, no. 14, pp. 13218-13224, Jul 2011. [Online]. Available: http: //www.opticsexpress.org/abstract.cfm?URI=oe-19-14-13218

[7] A. V. Smith and J. J. Smith, "Mode instability in high power fiber amplifiers," Opt. Express, vol. 19, no. 11, pp. 10180-10 192, May 2011. [Online]. Available: http://www.opticsexpress.org/abstract.cfm? URI $=$ oe- $19-11-10180$

[8] C. Jauregui, F. Stutzki, A. Tünnermann, and J. Limpert, "Thermal analysis of Yb-doped high-power fiber amplifiers with Al:P codoped cores," Opt. Express, vol. 26, no. 6, pp. 7614-7624, Mar 2018. [Online]. Available: http://www.opticsexpress.org/abstract.cfm? URI=oe-26-6-7614

[9] F. Beier, F. Möller, B. Sattler, J. Nold, A. Liem, C. Hupel, S. Kuhn, S. Hein, N. Haarlammert, T. Schreiber, R. Eberhardt, and A. Tünnermann, "Experimental investigations on the TMI thresholds of low-NA Yb-doped single-mode fibers," Opt. Lett., vol. 43, no. 6, pp. 1291-1294, Mar 2018. [Online]. Available: http://ol.osa.org/abstract.cfm?URI=ol-43-6-1291

[10] B. G. Ward, "Finite element steady periodic beam propagation analysis of mode instability in high power fiber amplifiers," Opt. Express, vol. 26, no. 13, pp. 16875-16883, Jun 2018. [Online]. Available: http://www.opticsexpress.org/abstract.cfm?URI=oe-26-13-16875

[11] H.-J. Otto, A. Klenke, C. Jauregui, F. Stutzki, J. Limpert, and A. Tünnermann, "Scaling the mode instability threshold with multicore fibers," Optics Letters, vol. 39, no. 9, pp. 2680-2683, May 2014.

[12] A. Klenke, M. Müller, H. Stark, F. Stutzki, C. Hupel, T. Schreiber, A. Tünnermann, and J. Limpert, "Coherently combined 16-channel multicore fiber laser system," Optics Letters, vol. 43, no. 7, pp. 15191522, Apr 2018.

[13] J. Ji, S. Raghuraman, X. Huang, J. Zang, D. Ho, Y. Zhou, Y. Benudiz, U. B. Ami, A. A. Ishaaya, and S. Yoo, "115 W fiber laser with an all solid-structure and a large-mode-area multicore fiber," Opt. Lett., vol. 43, no. 14, pp. 3369-3372, Jul 2018. [Online]. Available: http://ol.osa.org/abstract.cfm?URI=ol-43-14-3369

[14] E. Coscelli, R. Dauliat, F. Poli, D. Darwich, A. Cucinotta, S. Selleri, K. Schuster, A. Benoît, R. Jamier, P. Roy, and F. Salin, "Analysis of the Modal Content Into Large-Mode-Area Photonic Crystal Fibers Under Heat Load," IEEE Journal of Selected Topics in Quantum Electronics, vol. 22, no. 2, pp. 323-330, Mar 2016.

[15] K. R. Hansen, T. T. Alkeskjold, J. Broeng, and J. Lægsgaard, "Thermally induced mode coupling in rare-earth doped fiber amplifiers," Opt. Lett., vol. 37, no. 12, pp. 2382-2384, Jun 2012. [Online]. Available: http://ol.osa.org/abstract.cfm?URI=ol-37-12-2382

[16] L. Dong, "Stimulated thermal rayleigh scattering in optical fibers," Opt. Express, vol. 21, no. 3, pp. 2642-2656, Feb 2013. [Online]. Available: http://www.opticsexpress.org/abstract.cfm?URI=oe-21-3-2642

[17] J. Lægsgaard, F. Poli, A. Cucinotta, and S. Selleri, "Theory of thermooptic instabilities in dual-core fiber amplifiers," Opt. Lett., vol. 43, no. 19, pp. 4775-4778, Oct 12018.

[18] L. Rosa, H. Mckee, F. Poli, S. Selleri, L. Vincetti, and A. Cucinotta, "Thermally-driven mode coupling in multi-core optical fibers," in Advanced Photonics 2018 (BGPP, IPR, NP, NOMA, Sensors, Networks, SPPCom, SOF). Optical Society of America, 2018, p. JTu5A.79.
[Online]. Available: http://www.osapublishing.org/abstract.cfm?URI= SOF-2018-JTu5A.79

[19] G. P. Agrawal, Nonlinear Fiber Optics. San Diego: Academic Press, 2007.

[20] “Comsol Multiphysics ${ }^{\circledR}$ v. 5.0," www.comsol.com, COMSOL AB, Stockholm, Sweden.

[21] D. C. Brown and H. J. Hoffman, "Thermal, stress, and thermo-optic effects in high average power double-clad silica fiber lasers," IEEE Journal of Quantum Electronics, vol. 37, no. 2, pp. 207-217, Feb. 2001.

[22] A. Cucinotta, F. Poli, S. Selleri, L. Vincetti, and M. Zoboli, "Amplification Properties of $\mathrm{Er}^{3+}$-Doped Photonic Crystal Fibers," J. Lightwave Technol., vol. 21, no. 3, p. 782, Mar 2003. [Online]. Available: http://jlt.osa.org/abstract.cfm?URI=jlt-21-3-782

[23] C. Xia, M. A. Eftekhar, R. A. Correa, J. E. Antonio-Lopez, A. Schülzgen, D. Christodoulides, and G. Li, "Supermodes in Coupled Multi-Core Waveguide Structures," IEEE Journal of Selected Topics in Quantum Electronics, vol. 22, no. 2, pp. 196-207, Mar 2016.

[24] M. M. Jørgensen, S. R. Petersen, M. Laurila, J. Lægsgaard, and T. T. Alkeskjold, "Optimizing single mode robustness of the distributed modal filtering rod fiber amplifier," Opt. Express, vol. 20, no. 7, pp. 7263-7273, Mar 2012. [Online]. Available: http://www.opticsexpress.org/abstract.cfm?URI=oe-20-7-7263

[25] F. Poli, E. Coscelli, A. Cucinotta, S. Selleri, and F. Salin, "Single-mode propagation in yb-doped large mode area fibers with reduced cladding symmetry," IEEE Photonics Technology Letters, vol. 26, no. 24, pp. 2454-2457, Dec 2014. 